\title{
Method, Design and Implementation of a Multiuser Indoor Localization System with Concurrent Fault Detection
}

\author{
Fabio Veronese, Sara Comai, Matteo Matteucci, Fabio Salice \\ Politecnico di Milano \\ Department of Electronics, Informatics and Bioengineering \\ - Polo Regionale di Como - \\ Via Anzani 42, 22100, Como, Italy \\ $<$ name>.<surname>@polimi.it
}

\begin{abstract}
Thanks to the large diffusion of small wearable devices there are several systems designed for indoor localization. Among the proposed solutions, RF-based systems have been deeply investigated due to their flexibility and limited costs. When these systems are employed as assistive tools, they should be dependable, recognizing autonomously when they are affected by a fault. In this paper, we present a method to provide multiuser localization with concurrent fault detection. We focus on two possible sources of faults: natural hardware (e.g., empty battery, faulty components) and human-made (e.g., device not worn). The presented methodology relies on two independent measurement systems and on a model based fault detection apparatus, checking for discrepancies in the behavior of the subsystems. This method is implemented to provide dependable localization in fragile people (such as elderly or people with small impairments) dwellings. We present examples of simulations in a large environment, and an implemented case-study. The collected data confirm the validity of the approach.
\end{abstract}

\section{Keywords}

Human Indoor Localization; Home Automation; Assistive Technology; Smart Home; Dependability; Fault Detection; Human-Made Fault Detection.

\section{Categories and Subject Descriptors}

B.8.1 [PERFORMANCE AND RELIABILITY]: Reliability, Testing, and Fault-Tolerance; C.4 [PERFORMANCE OF SYSTEMS]: Reliability, availability, and serviceability

\section{INTRODUCTION}

Indoor human localization and tracking is a growing technology, boosted by the development of small, wearable, cost effective, low power devices. Localization systems are the basis for context-aware services, advertising, navigation and other commercial activities. This implies they are an important actor in Smart Cities and Smart

Permission to make digital or hard copies of all or part of this work for personal or classroom use is granted without fee provided that copies are not made or distributed for profit or commercial advantage and that copies bear this notice and the full citation on the first page. To copy otherwise, to republish, to post on servers or to redistribute to lists, requires prior specific permission and/or a fee. MOBIQUITOUS 2014, December 02-05, London, Great Britain Copyright (c) 2014 ICST 978-1-63190-039-6 DOI 10.4108/icst.mobiquitous.2014.258215
Environments, bounding real world to virtual spaces (social networks, websites, etc.).

Nonetheless Indoor Localization Systems are being widely used also as assistive tools in many environments. In these settings they are usually designed to support (or restore) independent life. One of the most important needs is certainly the need for security, which stands at the second level of Maslow's hierarchy [15], being highly important for all the human beings. This applies both to fragile people (such as elderly, people suffering from small impairments), who need their family to be watching over them, and to their families, who want to be sure that their loved ones are in safe conditions. An assistive system, able to share the position of a fragile person to his/her relatives, can play a significant role, being a tool to satisfy these needs. Moreover is not uncommon for fragile people living alone, to experience serious consequences due to domestic falls or accidents.

This work refers to Indoor Human Localization (IHL) systems for fragile people, where dependability features are needed and often other assistive systems are already deployed. The main idea is to exploit already-in-place sensors, as a source of redundant information about the person's position. Crossing this information with the one from the IHL system, through a model based analysis, enables to identify both systems faults. While most of the design effort for dependability is usually devoted to manage faults generated by components of the system (e.g., hardware or software, technically named natural faults), in this work we will focus also on those generated by the users, also called human-made faults. In this paper we present the method, the design and a reliable implementation of such IHL system, where natural and human-made faults can be detected.

\section{RELATED WORK}

In order to improve the reliability of localization systems, different approaches have been implemented. In this section first we will introduce indoor human localization techniques from the literature, then we will report the approaches carried on to improve reliability.

\subsection{Indoor Humans Localization}

Nowadays several technologies for IHL, based on various physical principles, are available. Torres-Solis et al. [26] classify IHL systems based on the measured quantity: photonic energy, sonic waves, mechanical energy, magnetic fields, atmospheric pressure and radio frequency (RF) waves. This last physical quantity is widely used, in cost-effective and flexible systems, by leveraging different methods. Focusing on RF-based methodologies, the main sampled quantities, observed to perform localization, are: Angle of Arrival, 
Time of Flight, Differences in Time of Arrival, Received Signal Strength (RSS) [26, 8, 11]. Depending on the observed quantity it is possible to adopt a method based on Lateration/Angulation (computing a precise position in space from three or more measurements of distance/angle from known locations [8]) or on Fingerprinting (defining specific areas in the environment and identifying the target presence in one of them [8]). Looking at different technologies inside the RF category, system can leverage Radio Frequency Identification (RFID), Wireless Local Area Network (WLAN), Bluetooth, Wireless Sensor Netwok (WSN), Ultra Wide Band (UWB), TV broadcasts (UHF) or mobile phone communications (UMTS, GPRS, etc.) [8]

\subsubsection{Sensor Fusion}

Wireless RF technologies, presented in the previous section, rely on an uncontrolled and highly shared medium: many possible happenings (e.g., interference, shadowing, multipath) inevitably lead to inaccuracies and errors. The most diffused approach to increase the accuracy of localization, is to leverage two or more physical quantities and combine them to obtain improvements both in precision and reliability [8]. Nonetheless, even if leveraged in less systems, Sensor Fusion can also provide a certain level of resilience to faults affecting one of the measure systems.

Dead reckoning systems, usually based on the integration of acceleration measurements coming from an IMU (Inertial Measurement Unit), are subject to estimation error accumulation: in most of the proposed approaches the system controls drift, either by considering other physical quantities such as RF [10], atmospheric pressure [22]) or relying on a priori knowledge [28] and landmark identification [27].

In other systems, RF and UltraSounds (US) are used together to improve the final precision, or to overcome environmental issues. The Cricket localization system [18] leverages the difference in time propagation between RF and US signals to estimate the distance to a known emitter. This enables the designers to implement a signal transmission protocol and a processing algorithm capable to ignore the data affected by noise (e.g., multipath, reflections), thus obtaining a better estimate than using RF or US independently.

A very similar approach is presented by YunFei et al. [17]: their system leverages the time difference between US and RF propagations to estimate the distance from a device with a known position. Furthermore, they include a signal integrity monitoring, leveraging measurement redundancy. This allows them to identify situations with inaccurate estimations and, in case, to send the user a feedback.

The positioning system reported by Do et al. [9] is based on three different technologies, crossing information coming from WLAN, GPS and cell towers. The system is deployed on a mobile terminal, where the data are collected, processed and the result is available to the user. The aim is to locate the user seamlessly, in indoor or outdoor environments, trying to rely on the most accurate measurements available. The system also comprises a communication policy between the terminal and the remote localization server, trying to minimize the requests when a good localization can be provided by the terminal itself

\subsubsection{Dependability in localization}

A dense literature has been developed about methods to exclude wrong measurements thanks to redundancy, when dealing with localization and lateration. Sturza developed a method in 1988 [25], and since then several techniques improved or leveraged those principles, but mainly in GPS (Global Positioning System) applications and outdoor environments. Three conventional versions of receiver autonomous integrity monitoring (RAIM) methods are the chi-square test, the horizontal protection level test (HPL) and the multi-hypothesis solution separation test (MHSS) [9].

Do et al. [9] used these techniques within an hybrid TV-GPSWLAN localization system, facing multi-faults conditions. The proposed solutions are the variants of the three algorithm listed before, modified in order to include an iterative fault detection and exclusion.

In indoor settings, anyway, there are few examples of works explicitly devoted to dependability. YunFei et al. [17] use the same principle (of redundancy) to identify faulty measurements. They keep trace of the estimation precision, looking for biases in a specific precision parity matrix. This is used not to exclude the measure from the estimation procedure, but to warn the user that the measurements are not reliable.

\section{SYSTEM DEFINITION AND MODELING}

Before going into methodology and implementation details it is necessary to introduce some definitions.

\subsection{Definitions}

The model of the system and the concepts of Fault, Error, Failure refer to the terminology proposed in Avižienis et al. [7]. In our work the system is an indoor entity, constituted by hardware, software and humans, interacting with the environment. The system has a structure made of interacting components, and a behavior describing what the system does to implement its function and can be represented as a set of states. Perceived by the users (e.g., humans, other systems, etc.), the behavior represents the service the system is providing.

\subsubsection{Fault, Error, Failure}

When we observe a deviation from at least one state in the system behavior with respect to the correct sequence of states, that deviation is an error. It is worth noting that potentially many errors may not be observable and may not cause a service failure: this could be due to temporary masking (the environment has not stimulated the system) or thanks to the application of a fault tolerance strategy. Any error has a cause, which is called fault. A fault first causes an error in an internal service state (i.e. a component of the system) but the observable state is not immediately affected. The error affects other system states, till the observable state is reached and the system service fails. A system service failure is when the delivered service deviates from the correct service, violating the functional specifications. In this work we exclude the possibility service failures due to inadequate or incomplete specifications, thus failures are caused by faults only.

In this work we assume that the development phase is fault-less, thus we will focus on operational faults (i.e. those occurring during the system's use phase). Furthermore, it is important to distinguish between natural faults, caused by natural phenomena, and humanmade faults, caused by human actions.

\subsection{System Structure}

The system is composed by the following components.

IHL subsystem: this system estimates the target people's positions in the indoor environment.

Anonymous Interaction Detection (AID) subsystem: a system able to provide localization events detected by ubiquitous (general purpose) sensors placed inside the house.

Fault Detection subsystem: Information gathered from both IHL and AID subsystems are collected by the fault detection subsystem, which is able to process them to detect when a fault condition is verified. 


\subsection{System Specification}

The mission of the presented system architecture is to provide dependable IHL. The case study is an indoor positioning system able to detect the position of one or more fragile persons inside a building. Caregivers are able to get the position of the monitored persons. Both inhabitants and caregivers are users, the service provided is the fragile people localization, to achieve "mutual reassurance" in an independent living framework.

\subsubsection{Functional Specifications}

The system provides the target people's positions. The localization targets must be inside a specific space (a part or the whole building).

\subsubsection{Non-functional Specifications}

The system implements a method for concurrent fault detection. In particular, this system evaluates its operational health during normal functionality, to detect a fault when it occurs. It is worth noting that the system is only able to detect faults, not to tolerate them. Thus, we can state the system is self-checking (and not faulttolerant). Furthermore the fault is detected, but its source is not generally identified.

\subsubsection{Other Requirements and Hypotheses}

After defining specifications, we introduce few other conditions and hypotheses:

- The development phase is flawless and does not introduce any fault.

- The IHL subsystem should have coverage on the whole indoor environment where the persons are tracked.

- Users do not temper the system components, nor act purposefully when their action causes a fault.

\subsection{Components Modeling}

When designing a self-checking system, a possible approach is to identify and describe all the possible faults and errors. This process, though, provides only a little flexibility and it is improbable for it to detect a fault not identified. An alternative approach can be designing a model-based self-checking system. Indeed, as stated by Isermann [13], it is possible to detect a fault by using the dependencies between different measurable signals; to this aim it is necessary to build a model of the dependency itself. Given the measured real-world quantities and a model, reproducing the expected system behavior, it is possible to generate features (e.g., states, parameters or residuals coming from the system model). If their values do not comply with the nominal characteristics of the system, a fault is detected [13]. This approach enables to build a description of error not only more flexible (being able to detect faults in a more general way), but also, in certain conditions, more synthetic. To exploit these advantages we preferred the model based approach.

Before modeling the components functionality, let us describe the environment. Since we are dealing with the indoor localization problem, we can define the space of interest (Home space) $H \subset \mathbb{R}^{2}$ (extendable to $\mathbb{R}^{3}$ ) as a subset of the whole space $\tilde{H}$ excluding $W \subset \tilde{H}$, the set of points inside walls and, more generally, in unreachable positions

\subsubsection{IHL model}

Once defined the environment we must provide a description of the IHL subsystem functionality. In the problem we have addressed, the localization system is supposed to provide the person position in the house space $H$. Since the aim of the system is to provide the person's position in the home environment, we can describe its output $\hat{\mathbf{x}}_{L}$ as:

$$
\hat{\mathbf{x}}_{L}=\mathbf{x}_{p}+\boldsymbol{\xi} \quad \mathbf{x}_{p}, \hat{\mathbf{x}}_{L} \in H \subset \mathbb{R}^{2}
$$

where $\mathbf{x}_{p}$ is the actual position of the person and the measured values are affected by an additive error $\xi$. It is possible to consider the output of the IHL system as a set of points $L \subset H$, surrounding the result position $\hat{\mathbf{x}}_{L}$ within a maximum distance $\delta_{t h}$, granting a certain precision (e.g., $t h=90 \%$ ). Obviously a person can not move through walls, and this means to exclude not only points belonging explicitly to walls, but also those which are behind a wall (i.e. in another room or outside the building), with respect to the estimated position $\hat{\mathbf{x}}_{L}$ :

$$
L \triangleq\left\{\mathbf{y} \in H:\left\|\mathbf{y}-\hat{\mathbf{x}}_{L}\right\| \leq \delta_{t h}, \overline{\mathbf{y}}_{L} \cap W=\varnothing\right\}
$$

Defined $L$, we can model IHL system fault through the condition:

$$
\mathbf{x}_{p} \notin L \Rightarrow \mathrm{F}_{L}
$$

which states that if the actual position of the person is not inside the result set $L$, a fault $\mathrm{F}_{L}$ is occurring.

Nonetheless, we have only an estimation of the person's position, the real world value $\mathbf{x}_{p}$ is unknown in such settings. If the aim is to provide dependability, verifying the estimation is correct, it is necessary to gather independent measurements of $\mathbf{x}_{p}$, for example deploying a second redundant IHL system. This, anyway, means also to double the costs, with the only advantage of dependability. Instead of deploying a redundant IHL system, we might leverage other systems, designed to provide another service, but still able to furnish the needed information about the person's position.

\subsubsection{Anonymous Interaction Detection (AID) model}

Potentially any element of a smart home environment with which ihabitants interact could be sensorized, so that a specific event can be recognized. This information (e.g., the usage of an hair drier, or the weight on a chair sit, the water tap opening/closing, the activity at a pc workstation, etc.) is not necessarily related to the functionality of the sensorized system, but still represents a localized event in time and space generated by a person. These events are in other words related to the real world person's position $\mathbf{x}_{p}$, which is necessary to identify IHL systems faults.

Generalizing and abstracting from the specific sensing technology, it is possible to identify a common model for these kinds of events. To keep the generalization valid, we must then accept the fact that the detected interaction might not be related to a specific person, thus let us define the detected presence as anonymous. First of all let us consider that each sensor $s \in S$ ( $S$ set of all sensors) has a known position in space $\mathbf{x}_{s} \in H$. Other inherent characteristics of the sensor are surely the interaction range $r_{s}$ (i.e., the maximum distance $\left\|\mathbf{x}_{p}-\mathbf{x}_{s}\right\|$ at which the activation event can take place), and a value $v_{s}$, referred to the sensor (e.g., the power consumption of the hair drier, the measured weight on the chair, the boolean status of the tap, the activity performed at the pc workstation). We can finally formalize this model by describing the sensor $s$ activation area $A_{s}$ as:

$$
A_{s} \triangleq\left\{\forall \mathbf{y} \in H,\left\|\mathbf{y}-\mathbf{x}_{s}\right\| \leq r_{s}, \overline{\mathbf{y x}}_{s} \cap W=\varnothing\right\}
$$

which includes also the condition against walls-crossing, as defined for IHL.

Furthermore, let us introduce $V_{s}$, the set of all the possible values (eventually an enumeration of discrete statuses or a range) of the sensor $s$ and $\bar{V}_{s}$ the set of those that are correlated to a presence (while dually $\underline{V}_{s}$ is the set of values read when no interaction has taken place):

$$
\forall s \in S, v_{s} \in \bar{V}_{s} \Rightarrow \mathbf{x}_{p} \in A_{s}
$$


Extending the single sensor case and considering a whole house, sensorized with a set of sensors $\mathcal{S}$, we can define the active area $\mathcal{A} \subseteq H$ for the whole AID system:

$$
\mathcal{A} \triangleq\left\{A_{s}: s \in \mathcal{S}, v_{s} \in \bar{V}_{s}\right\}
$$

Indeed it is necessary to consider also the possibility that a fault affects the AID system. Focusing only on the information related to $x_{r}$, we can define the fault of the AID system $\mathrm{F}_{A}$ as:

$$
\forall \mathbf{x}_{p} \in H, \exists s \in S:\left\{A_{s} \in \mathcal{A}, \mathbf{x}_{p} \notin A_{s}\right\} \Rightarrow \mathrm{F}_{A}
$$

which represents the condition in which the person is not in the activation area of a triggered sensor.

\subsubsection{Fault Detection}

With such modeling, it is possible to recognize that an AID system can provide an information similar to the one we could obtain by duplicating the IHL system. Thus, when an IHL and an AID systems are placed in the same environment $H$, the person must stand in an area identified concordantly by both the IHL and AID systems. Thus the following relation can be defined:

$$
\exists s \in S:\left\{A_{s} \in \mathcal{A} \wedge\left(L \cap A_{s}\right)=\varnothing\right\} \Rightarrow\left(\mathrm{F}_{L} \vee \mathrm{F}_{A}\right)
$$

Having identified an observable state, which implies the system behavior to deviate from the expected one, we can define it as an error E:

$$
\mathrm{E} \triangleq\left\{\exists s \in S: A_{s} \in \mathcal{A} \wedge\left(L \cap A_{s}\right)=\varnothing\right\}
$$

In case we have more people $p \in P$ to track simultaneously inside the house it is only necessary to consider all the $L_{p}$ areas:

$$
\mathrm{E} \triangleq\left\{\exists s \in S:\left\{A_{s} \in \mathcal{A} \wedge \forall p \in P,\left(L_{p} \cap A_{s}\right)=\varnothing\right\}\right\}
$$

\subsubsection{Sensors Limitations}

The models and the fault detection method introduced in the previous paragraphs have been designed to show a high generalization. Indeed, most of the localization and home ubiquitous systems can fit in those model, although, this brings also some drawbacks. Their outcome with respect to dependability is that it is not possible to grant the fault-secureness property[7] in any situation. In the following we will present the limitations that sensors introduce, and, where possible, provide a possible approach to mitigate their effects.

Natural interaction with home devices, appliances and interfaces, as we presented in the AID modeling, is related to the person's action in the environment. Obviously a person is not always interacting with the environment: This forces us to accept the condition that a person can be (and be localized) inside an area where no interaction takes place. This fact has a consequence on the system properties, since it makes impossible to guarantee the fault-secureness. For example if a fault of the IHL system occurs for the $i$-th target, as long as he/she does not interact with the AID system, no fault is identified.

Another possibility is that the "faulty" $i$-th target actually moves (its real world position $\mathbf{x}_{i}$, not $\hat{\mathbf{x}}_{i}$ ), close to the $j$-th target, who actually "shadows" the $i$-th target presence, by making the system consider any activation as made by $j$, even if actually made by $i$. Even if this might seem to limit the significance of the concurrent fault detection we want to put in place, it is important to remark that time period between two AID system events depends on the persons' activity and on the sensors type and dissemination. The higher the sensors density and the finer the granularity, the more precise is the spatial resolution of the events; the more active the person is, the more frequent the interaction events are.

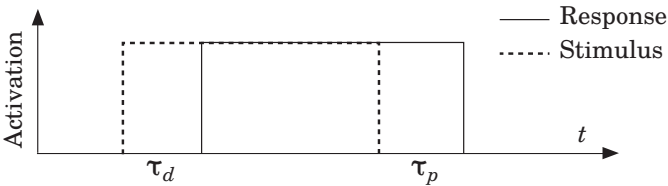

Figure 1: Sensor delay $\tau_{d}$ and persistance $\tau_{p}$ representation.

\subsubsection{Interaction versus Presence}

When needed, it is anyway possible to employ presence sensors. It is somehow similar to an IHL duplication, but a Presence Detection (PD) system might have a lower cost and/or other services/purposes. In that case the sensors activate not due to an interaction, but just passively because of the persons presence. If so we can extend what previously observed for interaction:

$$
\forall s \in S, \forall \mathbf{x}_{p} \in H \quad \mathbf{x}_{p} \in A_{s} \Leftrightarrow v_{s} \in \bar{V}_{s}
$$

In these conditions it is possible to consider not only (8), but also the dual condition:

$$
\begin{gathered}
\exists p \in P:\left\{\forall s \in S: A_{s} \in \mathcal{A} \vee\left(L_{p} \cap A_{s}\right)=\varnothing\right\} \Rightarrow \\
\Rightarrow\left(\mathrm{F}_{L} \vee \mathrm{F}_{A}\right)
\end{gathered}
$$

defining that any active area $L_{p}$ should have an intersection with at least an active sensor's area $A_{s}$, otherwise $\mathrm{F}_{L}$ or $\mathrm{F}_{A}$ occurs. The related error formulation is:

$$
\mathrm{E}^{\prime} \triangleq\left\{\exists p \in P:\left\{\forall s \in S: A_{s} \in \mathcal{A} \vee\left(L_{p} \cap A_{s}\right)=\varnothing\right\}\right\}
$$

\subsubsection{Temporal Formulation}

The formulation of the previous paragraphs has an instantaneous connotation. In real world though, there are also issues related to sensors temporal dynamics, which can affect significantly the behavior of the activation. This imposes the modeling to introduce a temporal connotation to evaluate properly their activation.

First of all not all the quantities are time-dependent. We have to introduce the time $t$ and:

$$
\begin{aligned}
& L_{p}(t) \triangleq\left\{\left.L_{p}\right|_{\hat{\mathbf{x}}_{p}=\hat{\mathbf{x}}_{p}(t)}\right\} \\
& \mathcal{A}(t) \triangleq\left\{A_{s}: s \in S, v_{s}(t) \in \bar{V}_{s}\right\}
\end{aligned}
$$

where $\hat{\mathbf{x}}_{p}(t)$ is the position of the $p$-th person estimated by the IHL system at time $t$, and $v_{s}(t)$ is the value of the $s$-th sensor at time $t$. Let us define the delay and the persistence of a sensor. The delay $\tau_{d}$ is the time, since the stimulus beginning the sensor needs to activate, the persistence $\tau_{p}$ is the time lapse during which the sensor remains active even without stimulus (Figure (1)).

An emblematic example of the temporal issues is represented by PIR sensors, but the principle can be applied to any presence sensor with non-instantaneous timing. They are sensors $s \in S_{P}$ triggering immediately for changes in position $\dot{\mathbf{x}}_{p}(t) \in A_{s}$, but they deactivate after $\tau_{p}$ seconds since a person has stopped (in their area of activation) or has left. Thus they have no delay, but persistence $\tau_{p}$ :

$$
\begin{aligned}
& \left\{\begin{array}{lll}
\dot{\mathbf{x}}_{p}(t) \neq 0 & t>t_{0} & \text { (a) } \\
\mathbf{x}_{p}(t) \in A_{s} & t_{0} \leq t<t_{1} & \text { (b) } \\
\mathbf{x}_{p}(t) \notin A_{s} & t \geq t_{1} & \text { (c) }
\end{array} \Rightarrow\right. \\
& \Rightarrow \begin{cases}A_{s} \in \mathcal{A}\left(t_{0}+\tau\right) & 0 \leq \tau \leq\left(t_{1}+\tau_{p}\right) \\
A_{s} \notin \mathcal{A}\left(t_{1}+\tau\right) & \tau>\tau_{p}\end{cases}
\end{aligned}
$$

which models the behavior of a person in movement (clause (15.a)), inside the area of the $s$-th sensor from $t_{0}$ to $t_{1}$ (15.b) and exiting after (15.c). 
Furthermore, to model a person stopping at time $t_{2}$ (16.b-c), inside the area $A_{s}$ (16.a); we can add the following:

$$
\begin{aligned}
& \left\{\begin{array}{lll}
\mathbf{x}_{p}(t) \in A_{s} & \forall t & \text { (a) } \\
\dot{\mathbf{x}}_{p}(t) \neq 0 & t<t_{2} & \text { (b) } \\
\dot{\mathbf{x}}_{p}(t)=0 & t \geq t_{2} & \text { (c) }
\end{array} \Rightarrow\right. \\
& \Rightarrow \begin{cases}A_{s} \in \mathcal{A}\left(t_{2}+\tau\right) & 0 \leq \tau \leq \tau_{p} \\
A_{s} \notin \mathcal{A}\left(t_{2}+\tau\right) & \tau>\tau_{p}\end{cases}
\end{aligned}
$$

Given (15-16) it is possible to state that, in an environment fully covered by PIR sensors, a person is surely located inside the area of the last active PIR sensor which contained him/her. Indeed if a person moves from an area $A_{s}$ to another, $\dot{\mathbf{x}}_{p}(t)$ is not null, activating the sensors. Finally, we can state that, if the person $p$ 's last active PIR area $A_{s p}(t)$ and the current location area $L_{p}(t)$ have no intersection, a fault has occurred:

$$
\exists p \in P:\left\{A_{s p}(t) \cap L_{p}(t)=\varnothing\right\} \Rightarrow\left(\mathrm{F}_{L} \vee \mathrm{F}_{A}\right)
$$

Similarly to previously defined errors/faults, we can identify the error $\mathrm{E}_{\mathrm{PIR}}^{\prime}$ :

$$
\mathrm{E}_{\mathrm{PIR}}^{\prime} \triangleq\left\{\exists p \in P: A_{s p}(t) \cap L_{p}(t)=\varnothing\right\}
$$

While, on the other hand, Equation (10) must be modified in order to consider the sensor's persistency:

$$
\begin{aligned}
& \mathrm{E}_{\mathrm{PIR}} \triangleq\left\{\exists s \in S_{P}: A_{s} \in \mathcal{A}(t) \wedge\right. \\
& \left.\wedge A_{s} \cap L_{p}(t-\tau)=\varnothing, 0 \leq \tau \leq \tau_{p}\right\}
\end{aligned}
$$

Formulations similar to this can be described for other sensors. In the following, we will leverage this PIR sensors modeling to detect faults in experimental settings.

\subsection{Fault Observability}

As introduced in the definition section, faults have two main phases of existence: in the first they have already happened, but their effects are not observable yet. Only when a specific situation verifies then the fault becomes observable as an error, it is stimulated by the system conditions. In technical terms, a fault remains masked, and it is not detectable, until the output of the system remains compliant with the specifications: no error can be observed. In many situations, the system can be object of a self-test to verify if error can be detected, but this is not applicable to IHL. Indeed it is not very reasonable to require the user to move in a specific place periodically, to perform a kind of self-test. This remark implies that, in the scenario of this work, it is necessary to wait for the opportune situation for an error to be observable, and thus for a fault to be detected.

\section{CASE STUDY}

As presented in the introduction, the aim of this work is to provide a dependable localization system inside the home environment of people with special needs, in order to help them, to feel more secure and confident, and their family, to feel reassured about their beloved. This introduces a set of strict needs to be respected while designing the system.

\subsection{User's Need Centered Design}

When designing and implementing such systems, costs are considered extremely important, as well as installation effort and maintenance. A very precise and extensive system, with a price too high to be affordable by the user is useless. The more services is possible to provide with the same hardware, the better. Furthermore the system must be accepted by the host person, especially in terms of not feeling invaded by unpleasant devices. This can be respected by preferring wireless technology, non visual sensors, small and/or not visible devices. This philosophy drove our decisions, keeping our aim to build best effort useful system, rather than a high-performance one.

In this paper, we considered a possible implementation of the whole system. Its components are an RF localization system for healthcare indoor environment named LAURA, an off-the-shelf modular wireless Home Automation (HA) system, Z-wave, and a Fault Detection apparatus based on Esper. In these sections we describe the characteristics of all the subsystems, we present the technology chosen and the reasons which drove the decision.

\subsection{The Indoor RF Localization Subsystem}

LAURA $[20,21]$ is a localization system designed for people tracking in indoor environments. It is based on a $2.4 \mathrm{GHz} \mathrm{WSN}$, with a specifically designed addressing protocol. Originally developed by Lim et al. [14], the localization method relies on the RSSI between a mobile node of the WSN and the other location-known fixed nodes (anchors). It takes advantage of a dynamic and adaptative calibration by considering the RSS measurements also among fixed anchors. The raw estimation is then processed through a particle filter, which uses the given distances to solve the lateration problem, to smooth the output and to avoid non consistent movements and non permitted paths (wall crossing).

\subsubsection{Subsystem Characteristics}

As briefly introduced, the LAURA system has several features making it an ideal candidate for our settings: it is a critical mission assistive system, easily deployable, wireless, battery powered and no configuration is needed, making it particularly suitable for home environment. However, in the setting presented by Redondi et al. [20,21], it still lacks any method to provide dependability.

\subsection{The Home Automation Subsystem}

Ambient Assisted Living (AAL) and Home Automation (HA) technologies are nowadays spreading in our cities, changing our houses into smart homes. This not only brings the comfort and the services of a pervasive home control, but also provides a great opportunity to monitor and assist fragile people in their homes. The market of such products is highy competitive, and several standards, technologies, companies and solutions are nowadays available. Choosing the right solution is far from trivial as several factors are involved in the choice: ease of deployment, invasiveness, interoperability, costs, data rate, network topology, network size and communication medium. Nonetheless the investment is clearly a long-term one, so also the chances of survival of the system in the market, as pointed by Saidinejad et al. [23], should be taken into account.

\subsubsection{HA Systems Analysis and Choice}

The main WHANs (Wireless Home Automation Networks) available nowadays on the market are: Z-wave [4], ZigBee, 6LowPAN, Insteon, Wavenis, EnOcean [1] and MiWi [12, 19]. Most important Home Automation Networks (HAN) based on fieldbus technology are KNX [3] (successor of EIB), BACnet, LonWorks, X10, Profibus, Modbus, CANOpen, Universal Powerline Bus, CEBus, C-Bus and 1-Wire [23]. Their networks are mainly relying on twisted pair or power line medium. Some of them, like KNX and X10, have been specifically designed for residential environment, while others have general usage, both in industry and HA [23].

Saidinejad et al. [23] present an iterative method for the choice of suitable HA technology, given a set of constraints, considering also 
financial aspects. They report a case study, applying their approach while deciding which technology to adopt for the implementation of a smart home for elderly people. The application of their approach to a more general setting of a reliable system, providing not-invasive AAL and home monitoring, identified Z-wave and 6LowPAN as good candidates for our case study. Between them we adopted Z-wave, for the lower costs and its orientation toward home environments.

\subsubsection{Sensor Types}

Z-wave [4] has a very large set of different functional devices (both sensors and actuators) available for customers, providing different choices of manufacturers even for the same device type. To provide an implementation of two sensor types we selected PIR (Passive InfraRed) sensors and Door/Window contact sensors.

The PIR sensor detects the motion of a human in a range up to $5 \mathrm{~m}$, with a view angle of $360^{\circ}$, when ceiling mounted, or a range up to $10 \mathrm{~m}$ and an angle of $110^{\circ}$, when wall mounted. These different mountings are possible thanks to a replaceable lens, with two different shapes specifically provided for each setting. The returned PIR variable (activity) becomes true as soon as a person's movement is detected, with a persistence of $10 \mathrm{~s}$.

The Door/Window contact sensor (or simply DWS), has a very common design: it is based on a magnet, triggering a corresponding reed switch inside the active component of the device. The presence of a person can be inferred when the sensor status toggles.

\subsection{Fault Detection Apparatus}

The information collected from the two subsystems is numerical and has a strong temporal connotation. Time is important both in absolute (instantaneously) and in term of sequence (of events). This kind of data stream is not extraordinary complex, nor has the gigantic size that usually characterizes Streams of Complex Event [24], still the processing engine needs a certain flexibility to implement the introduced model. Furthermore, we want our approach to be potentially applied or extended to more complex settings (e.g., higher sampling frequencies, devices and/or subsystems number, problem complexity). To comply with all these requirements, among the available Complex Event Processing (CEP) Systems we identified Esper [2] as a candidate for our study.

\subsubsection{Esper}

Esper is an open source event series analysis and event correlation engine [2], able to recognize rich situations in event series and to trigger custom actions. Esper is designed for high volume event processing, where millions of events coming in would be impossible to record using classical database architectures. Esper provides a rich Event Processing Language (EPL) to express filtering, aggregation, and joins, possibly over sliding windows of multiple event series. It also includes pattern semantics to express complex temporal causality among events (followed-by relationship). One of the most interesting features is the possibility to use Plain Old Java Objects (POJOs) almost anywhere during the processing [2]. In our setting, we have adopted POJO to represent the system model and EPL queries to implement the Fault Detection apparatus.

\subsection{Case Study Specific Requirements}

In order to be modeled through the proposed method the case study system must comply with the following requirements:

Known Sensorized Inhabitant(s): Any person inside the environment is known since he/she wears an active localization device. The device is meant to be worn. Anyway, it is possible to remove it to avoid damages (e.g., under the shower), keeping it in a range of $2 \mathrm{~m}$.
Device Coverage: IHL and PIR sensors must cover the whole accessible area. Overlapping sensor areas are permitted.

Model Application: DWS are suitable for instantaneous error definition (10), while to PIR sensors can be applied $(18,19)$. The final error is the logic and of all of them.

\subsection{Faults Scenarios (FS)}

We can define separately Human-made Faults Scenarios (HFS) and Natural Fault Scenarios (NFS). The most important HFS are: (a) the inhabitant is not wearing the localization device; (b) the inhabitant damages the device.

NFS are related to devices and components wearing, etc., we can report few examples such as: (c) A device (HA or IHL) is not reachable (e.g., out of the WSN range); (d) A sensor (HA or IHL) is defective or malicious; (e) The localization mobile device cannot reach enough anchors to perform a valid localization; (f) A device (HA or IHL) runs out of power.

Furthermore both the IHL and the HA subsystems are able to provide specific information used to detect simpler fault conditions: $\mathrm{E}_{L 1}$ - An anchor device is not reachable anymore.

$\mathrm{E}_{L 2}$ - Not enough anchors are detected.

$\mathrm{E}_{L 3}$ - The user device is not reachable.

$\mathrm{E}_{Z 1}$ - Device has run out of power.

$\mathrm{E}_{Z 2}$ - Device is not reachable.

These more common (and simpler) errors are managed by the systems in a different and more traditional way, and, being not related to the model based methodology introduced with this work, they will not be further tested.

\subsection{Limitations}

Considering the application scenario, a home, it is worth to remark that the users population is considered sparse enough to avoid excessive fault masking. Indeed exploiting PIR sensors, it is possible to respect the privacy of the user. Nonetheless they are characterized by a wide activation area and the impossibility to identify even the number of detected humans: in densely populated environment this would result in almost continuous sensors activity, making impossible to extract any information. Anyway crowded and densely populated environment do not belong to our case study, involving home environment.

\section{EXPERIMENTS}

To test the presented approach we implemented the whole system and set a specific indoor area. After the data collection the performances of the system were evaluated.

\subsection{Environment}

The tests were held in some rooms of a Politecnico di Milano building in Como. As illustrated in Figure 2, nineteen fixed LAURA anchors and $7 \mathrm{Z}$-wave devices were distributed along the walls of a portion of one floor. One device was worn by a tester simulating the inhabitant, to track his movements.

\subsubsection{LAURA Configuration}

In order to setup LAURA, we used the IEEE 802.15.4 2.4GHz frequency band on channel 25, which is Wi-Fi free, preventing unwanted radio interference between Wi-Fi devices and WSN nodes. Each device was configured to transmit at a relative power of $-7 \mathrm{dBm}$. This power demonstrated to achieve acceptable results in terms of accuracy and precision, preventing unstable connections among nearby anchors. 


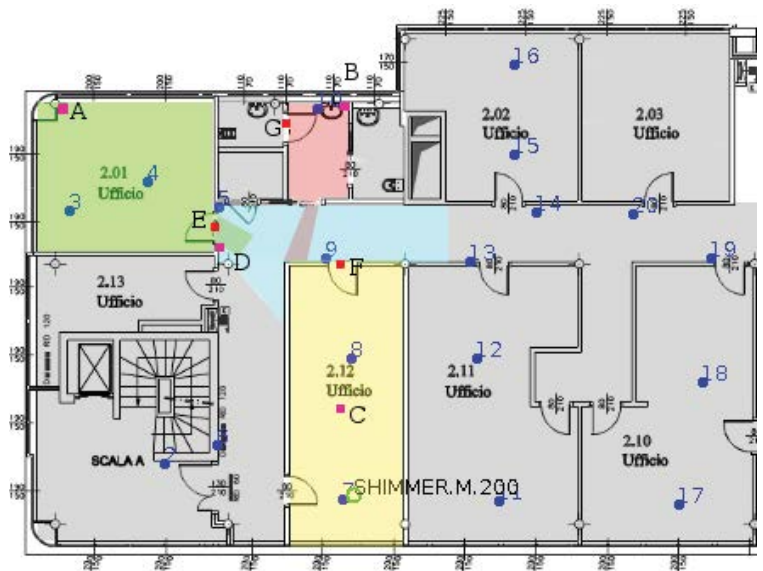

Figure 2: Testing environment and deployed sensors. In the map are visible the test areas and rooms (colored ones), LAURA fixed devices (blue numbered dots) and Z-wave devices (labeled with capital letters). PIRs (A-D) have corresponding colored activation areas.

\subsubsection{Z-wave Configuration}

We used two different categories of Z-wave devices in our environment. In order to detect human presence, four PIRs were mounted in three rooms and one corridor. Three DWSs were mounted on top of doors to monitor their states. Data gathered through these sensors were transmitted to the HA system.

The activation, which were stored on the Z-wave controller, were fetched via an ad hoc developed module, named "LEO", through standard HTTP calls over the Ethernet network. Aiming to be synchronized with LAURA, sensors' states were retrieved with a frequency of $1 \mathrm{~Hz}$.

\subsubsection{Model Parameters}

The model described in the previous sections can be adapted to the subsystem by tuning a specific set of parameters. In our settings the localization error of LAURA was studied, determining the value of $\delta_{t h}=3 \mathrm{~m}$, which is respected in the $84 \%$ of the estimations, as reported in Figure 3.

Concerning HA sensors, the values of maximum range provided by the producers of each device were reduced to the $80 \%$ : wall mounted PIRs had a maximum detection distance of $10 \mathrm{~m}$, thus

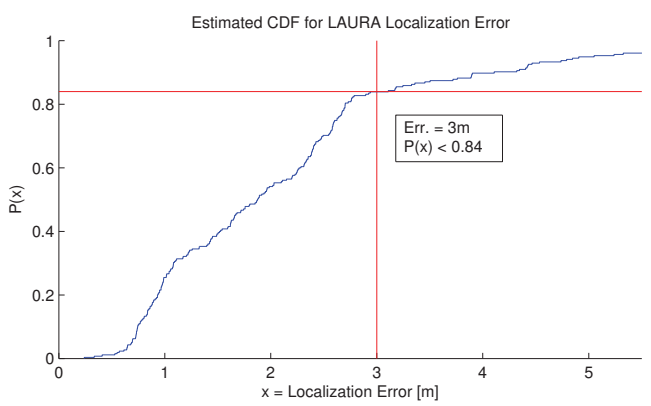

Figure 3: Estimated Cumulative Density Function (ECDF) for LAURA localization error. Tests performed to investigate the accuracy of the LAURA system resulted in the curve here depicted. The reported valued of $3 \mathrm{~m}$ bounds the $84 \%$ of the estimations.

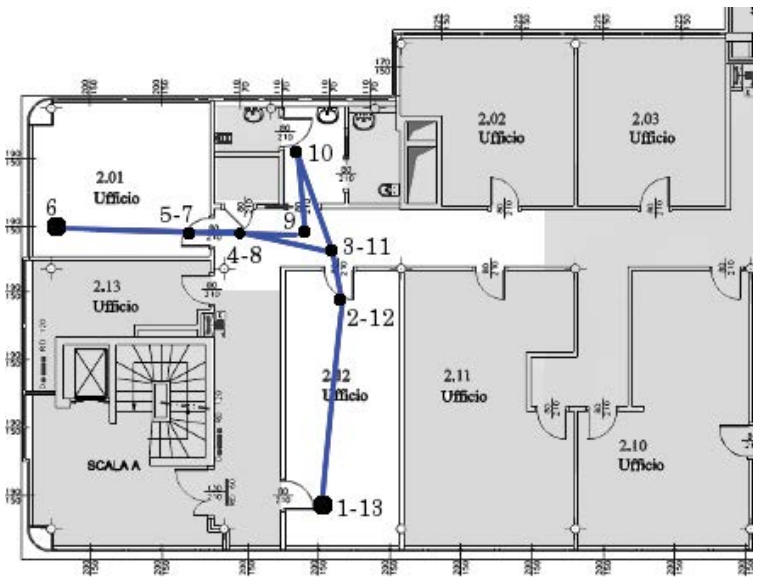

Figure 4: Path followed by the testers during experimental acquisitions. The white areas represent the sensorized space.

$r_{i \in P}=8 \mathrm{~m}$, similarly ceiling mounted ones had a reported range of $5 \mathrm{~m}$, which were reduced to $r_{i \in P}=4 \mathrm{~m}$. Considering doors, activation areas were assumed to be defined by a radius $r_{i \in \mathcal{D}}=1.5 \mathrm{~m}$.

\subsection{Test Protocol}

In order to obtain coherent and accurate data, both LAURA and the Z-wave system had to be synchronized during acquisition period. Since the sampling frequency is low, we used the Network Time Protocol (NTP) to synchronize clocks over Internet, assuring a precision in the order of $100 \mathrm{~ms}$ [16].

As illustrated in Figure 4, the trajectory for the path was polygonal, connecting predefined points by rectilinear segments. The inhabitant walked along the predefined path (designed to trigger the available sensors), with a constant speed. The tester stopped at each corner, before changing the direction: arrival and departure times at each point were recorded, in order to reconstruct the instantaneous position of the person $\mathbf{x}_{p}$. Concerning other environment elements, no constraint was defined, but changes were annotated. Since PIRs have time limitations, as mentioned previously in (Sensor Types), two lingering zones were added, where the tester remained still for a while in order to be undetected by the PIRs, highlighted in Figure 4 by greater dots (identified by numbers $1-13$ and 6 ). Time and duration of each single activity performed by the inhabitant and of any significant environment change, were annotated. The average test duration was about $3 \mathrm{~min}$, the overall duration of the tests was approximately $18 \mathrm{~min}$.

In order to model faults in the environment, two different policies were applied: Forgotten Device: A fault case was simulated by forgetting the worn device in a predefined location, while the tester continued its trajectory around the environment, triggering PIRs and DWSs. Blinded PIR: A PIR sensor was blinded in order not to detect the user, although it was active, charged and present on the network.

\subsection{Experimental Results}

The first run of experiments were in fault-free conditions. The collected data revealed no significant fault detection. As reported in Figure 6a, an initial error condition is risen and few short-duration error are visible (see first line in the figure). The initial fault is detected due to the HA system initialization: no sensor has been activated yet. While the brief fault detections (e.g., around $t=120 \mathrm{~s}$ ) are due to localization inaccuracies, as revealed comparing the actual position and the estimated position. Since we accepted $p=84 \%$ 


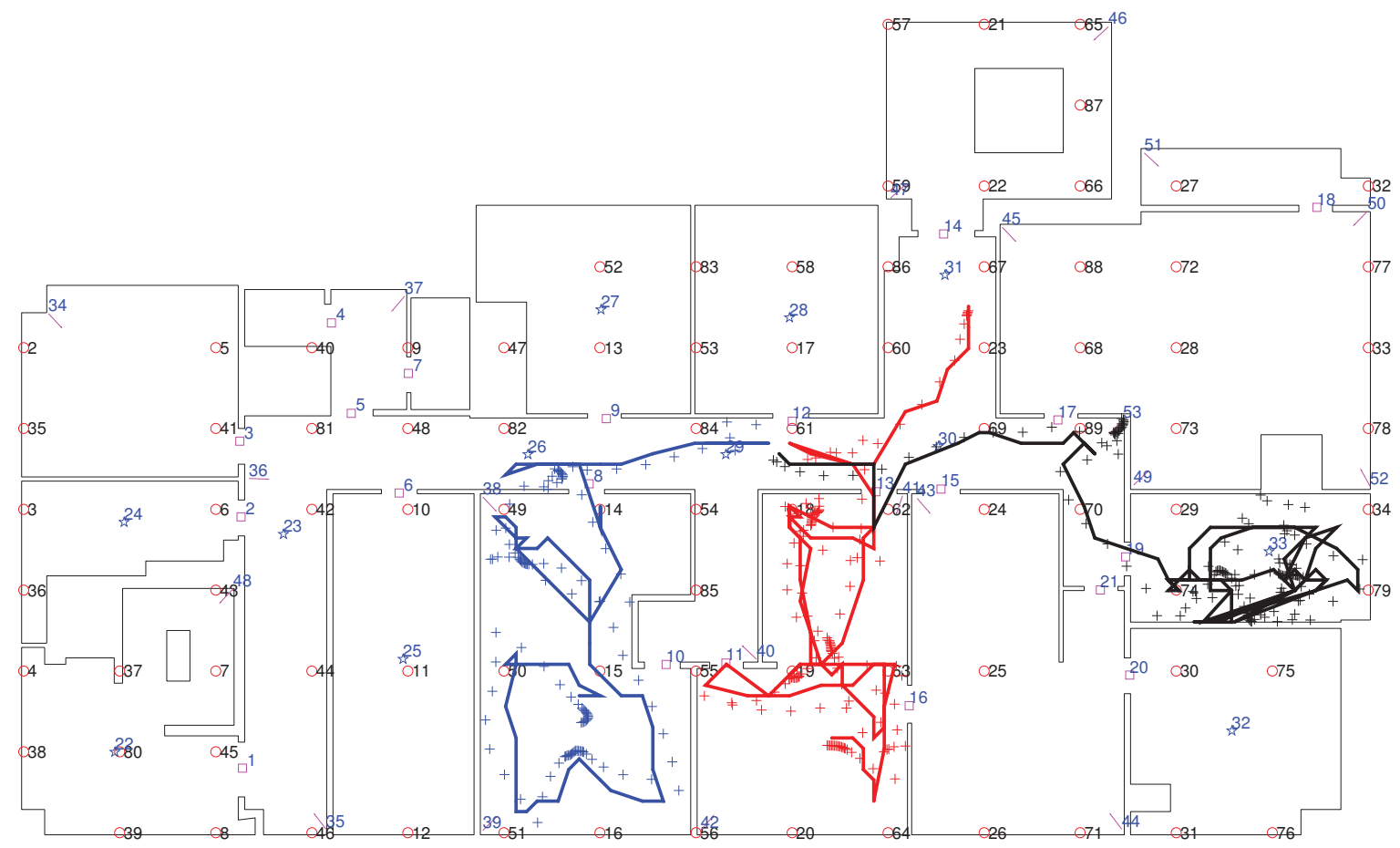

Figure 5: Simulation Environment: This map reproduces the whole floor where the experiments took place. The virtual sensorization is extended: red circles (labeled in black) represent localization anchors, squares are door sensors, stars ceiling-mounted PIR sensors, arrows wall mounted PIR sensors. Three virtual users trajectory are depicted: crosses $(+)$ represent the real position, tracks the position estimated by the IHL system.

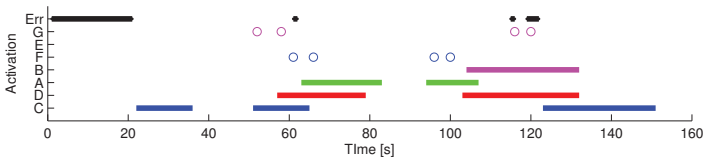

(a) Fault-free conditions. The brief and instantaneous fault activations are due to IHL system inaccuracies, which is concordant to the $84 \%$ precision threshold imposed.

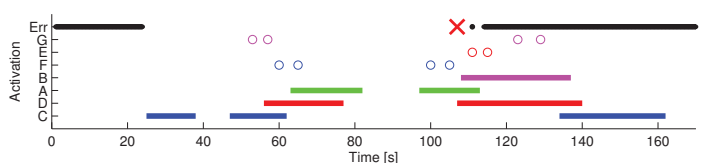

(b) Forgotten device. The device is left (X marker) in position 6 (Figure 4) along the trajectory.

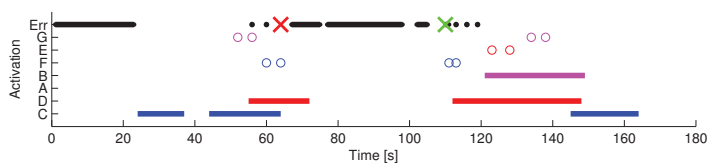

(c) Blind PIR sensor C. As the tester enters the room (leftmost X, point 5-7 in Figure 4) the Error is risen, then dismissed, as he exits (rightmost $\mathrm{X})$. Sensor's C green activation, present in Fig.(6a-6b), here is missing.

Figure 6: Real world experiments, user moving along the trajectory in Figure 4: Fault-free, Forgotten Device, Blind PIR. Lines represent PIR sensors activity, circles interactions with DWS, y axes letters refer to sensors as in Figure 2. when defining $\delta_{t h}$, it is possible that the estimated position exits the defined region: anyway, in those cases, the fault has punctual duration (up to $5 \mathrm{~s}$ ), can be identified or neglected. When we emulated the forgotten device condition, the system result was an expected fault detection. As visible in Figure $6 \mathrm{~b}$ as the inhabitant moves away from the device, left in position 6 (Figure 4) and activates other sensors, the fault detection response is sharp and stable. Second, if we blind a sensor, the system result is again an evident fault condition. As visible in Figure 6c we blinded PIR A (Figure 2): as the inhabitant enters the room (path from 5 to 7 in Figure 4), the fault is detected. In this conditions the fault detection is less sharp due to the same inaccuracies in localization, as already explained for fault-free conditions.

\subsection{Multiuser Simulation}

Due to the lack of available sensors and devices, we took advantage of simulation to provide multiuser highly sensorized environment tests. The environment is the reproduction of the one chosen for the experiments, considering the whole floor $\left(\mathrm{ca} .450 \mathrm{~m}^{2}\right.$ ) and adding users, as visible in Figure 5. The reference persons' positions have been generated thanks to a simple walk model, generating a continuous smooth acceleration and deceleration profile (maximum speed $0.7 \mathrm{~m} / \mathrm{s}$ ), with a smooth random steering policy. Collisions with walls are avoided by re-computing steering when needed, while periodically the agent lingers in a location (simulating the human behavior).

The IHL subsystem service was replicated by generating RF signal with the following model (being $d$ the distance and \# $w$ the number 


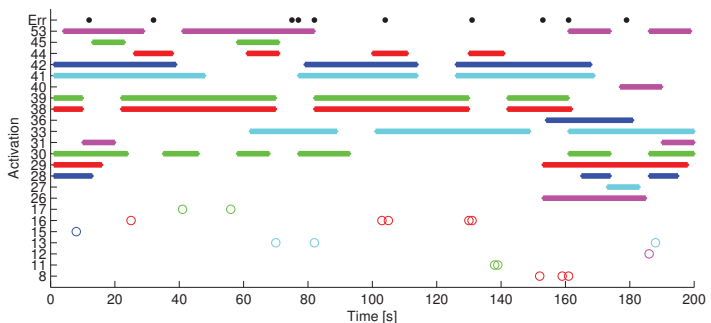

(a) Fault-free conditions: few punctual faults are detected, due to localization inaccuracies.

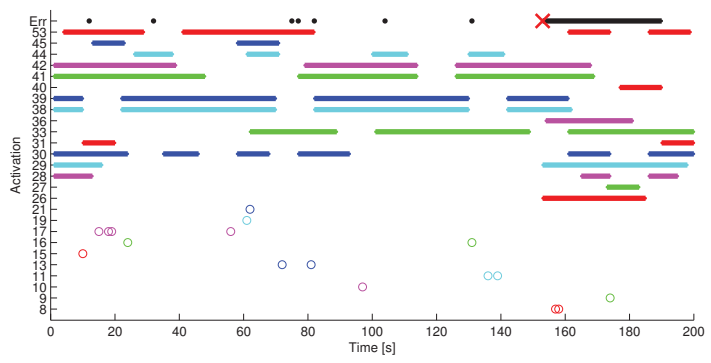

(b) Forgotten device: the leftmost user in Figure 5 leaves the localization device at 100s. As he triggers the device 26 (X marker) the fault (top black line) is detected.

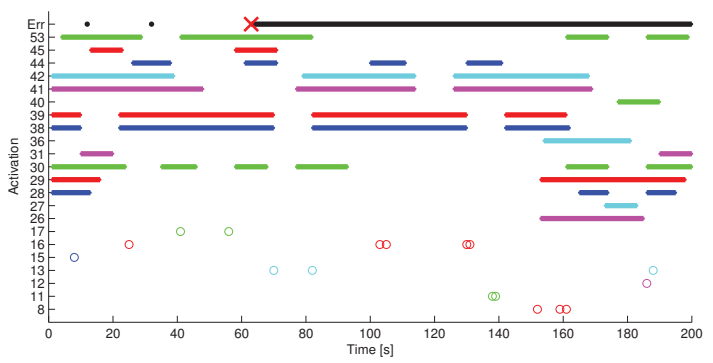

(c) Blinded PIR sensor 33. When the rightmost user in Figure 5 enters the room ( $\mathrm{X}$ marker), the fault is detected (top line).

Figure 7: Simulation results examples: fault-free, forgotten device and blinded PIR conditions. Three subjects are moving in the space, as showed in Figure 5. Lines represent PIR sensors activity, circles interactions with DWS, $y$ axes letters refer to sensors as in Figure 5.

of walls crossed by the signal from the emitter to the receiver):

$$
S=S_{0}-\left(\alpha \log _{10}\left(d / d_{0}\right)+\gamma(\# w)+\epsilon\right)
$$

and providing them to the LAURA system. The other parameters were $\alpha=3$, since we are in an indoor environment[20], and $\gamma=3 \mathrm{dBm}$, $d_{0}=0.04 \mathrm{~m}, S_{0}=-30 \mathrm{dBm}, \epsilon= \pm 2 \mathrm{dBm}$, as inferred from experimental measurements. To make it applicable to simulations, we also re-estimated the parameter $\delta_{t h}=2 \mathrm{~m}$.

Finally, the HA system behavior was reproduced, having the reference position of the persons, by implementing the sensors response, as presented by the manufacturers (introduced in the Z-wave subsystem section). In the simulated environment we exploited PIR sensors and DWSs. Concerning the interaction with DWSs, the agent entering the activation area of one of such devices had a uniform probability (of the 20\%) to interact with it, with a limit of two events (e.g., door opening and close).

Similarly to to real world experiments experiments, we ran simu-
Table 1: Simulation results. Test types: FF - fault free, FD - forgotten device, BS - blinded sensor; Results: (TP) true positives, (FP) false positives, (TN) true negatives, (FN) false negatives (Se) sensitivity, (Sp) specificity. The presence of faults during FF runs is due to localization results showing an error larger the imposed precision threshold $\delta_{t h}=2 \mathrm{~m}$.

\begin{tabular}{|c|c|c|c|c||c||c|c|}
\hline Test & TP & FP & TN & FN & TOT & Se & Sp \\
\hline FF & 2 & 55 & 1427 & 16 & 1500 & - & $96.28 \%$ \\
BS & 1392 & 0 & 0 & 108 & 1500 & $92.80 \%$ & - \\
FD & 550 & 176 & 717 & 57 & 1500 & $90.61 \%$ & $80.29 \%$ \\
\hline TOT & 1944 & 231 & 2144 & 181 & 4500 & $91.48 \%$ & $90.27 \%$ \\
\hline
\end{tabular}

lations in fault-free, in Blinded PIR and in Forgotten Device conditions. Three examples of simulation results are shown in Figures 7a, $7 \mathrm{~b}$ and $7 \mathrm{c}$, displaying the system behavior. The overall testing included 4500s of three simulated users wandering simultaneously and pseudo-randomly in the environment. The dataset was composed by 15 runs, lasting 5 minutes each, concerning fault-free conditions, Blinded PIR and Forgotten Device in equal parts. Concerning the Blinded PIR runs, faults were injected by making a PIR sensor always off during the run. The sensor was chosen in order to generate a fault, thus among those activated during the run. For each Forgotten Device run, instead, the position of a random localized device was fixed after 100s, still letting the agent trigger HA devices. The system performance was evaluated sample-by-sample (at 1Hz), considering each available environment state containing an observable fault, a positive trial. Considering only situations actually leading to errors, the experiments included 2125 faulty samples. The produced results are reported in Table 1: the system showed overall sensitivity of $91.48 \%$ and specificity of $90.27 \%$.

\section{CONCLUSIONS}

In this work we presented the method, design and implementation of a dependable IHL system, capable of concurrent fault detection. The system relies on two independent subsystems, whose result data are jointly checked by a fault detection apparatus. The proposed method is based on the definition of a model representing each of the two subsystems, and defining joint consistency conditions. The validity of the approach is proved applying it to a case study. The chosen case study subsystems are: LAURA localization system and a Z-wave based HA.

The obtained experimental results showed the validity of our approach, correctly reporting errors in fault-free and fault injected conditions. Furthermore, we generated multiuser data, creating them based on the knowledge of the environment and the systems. Results of multiuser simulations show the system correctly detecting faults also in case of several targets. Both specificity and sensitivity above $90 \%$ represent a satisfying performance. Nonetheless, if the application requires it, system model parameters can be tuned to benefit selectively sensitivity or specificity. Concluding, our approach, even under some limitations in terms of fault observability, enables the dependable localization of a set of persons inside an instrumented house, detecting both natural and human-made faults.

\section{FUTURE WORK}

Further tests will be performed in broader controlled environments, with more sensors in multiuser conditions. Moreover, a larger experimental setup will enable more detailed evaluations of fault stimulation and masking dynamics. It will be interesting to investigate the system behavior also in an actual home environment. 
As introduced in Sensor Types section, also other sensors might be modeled and leveraged into this system. A further development of the work presented hereby can consider complex patterns both in activations of HA sensors and in trajectories described by the IHL system. This will enrich the information, potentially extending faults even to specific detectable behaviors of the inhabitant.

The current method is able to extract a set of possibilities correlated with the identified error but it is currently impossible to identify exactly where the fault is (both human-made or natural). Future works will be focused on the correlation between a set of temporal sequences (system tests) and the faulty component. The approach we have in mind will be based on Amati et al.[6,5]. The main problem will be the real possibility to collect enough data to isolate the fault.

\section{REFERENCES}

[1] Enocean alliance. http://www.enocean-alliance.org/, 2013.

[2] Espertech official website. http://www.espertech.com, 2013.

[3] Knx association official website. http://www.knx.org/, 2013.

[4] Z-wave alliance official website. http://www.Z-wavealliance.org/, 2013.

[5] L. Amati, C. Bolchini, L. Frigerio, F. Salice, B. Eklow, A. Suvatne, E. Brambilla, F. Franzoso, and M. Martin. An incremental approach to functional diagnosis. In Defect and Fault Tolerance in VLSI Systems, 2009. DFT'09. 24th IEEE International Symposium on, pages 392-400. IEEE, 2009.

[6] L. Amati, C. Bolchini, F. Salice, and F. Franzoso. Improving fault diagnosis accuracy by automatic test set modification. In Test Conference (ITC), 2010 IEEE International, pages 1-8. IEEE, 2010

[7] A. Avizienis, J.-C. Laprie, B. Randell, and C. Landwehr. Basic concepts and taxonomy of dependable and secure computing. Dependable and Secure Computing, IEEE Transactions on, 1(1):11-33, 2004.

[8] G. Deak, K. Curran, and J. Condell. A survey of active and passive indoor localisation systems. Computer Communications, 2012.

[9] J.-Y. Do, M. Rabinowitz, and P. Enge. Multi-fault tolerant raim algorithm for hybrid gps/tv positioning. In Proc. ION Institute of Navigation National Technical Meeting, 2001.

[10] F. Evennou and F. Marx. Advanced integration of wifi and inertial navigation systems for indoor mobile positioning. Eurasip journal on applied signal processing, 2006:164-164, 2006.

[11] F. Franceschini, M. Galetto, D. Maisano, and L. Mastrogiacomo. A review of localization algorithms for distributed wireless sensor networks in manufacturing. International Journal of Computer Integrated Manufacturing, 22(7):698-716, 2009

[12] C. Gomez and J. Paradells. Wireless home automation networks: A survey of architectures and technologies. Communications Magazine, IEEE, 48(6):92-101, 2010.

[13] R. Isermann. Model-based fault-detection and diagnosis-status and applications. Annual Reviews in control, 29(1):71-85, 2005.
[14] H. Lim, L.-C. Kung, J. C. Hou, and H. Luo. Zero-configuration indoor localization over ieee 802.11 wireless infrastructure. Wireless Networks, 16(2):405-420, 2010.

[15] A. Maslow. Motivation and personality., 1954.

[16] D. L. Mills. Ntp performance analysis. University of Delaware, Newark, Del, USA, 2004.

[17] J. Park, M. Choi, Y. Zu, and J. Lee. Indoor localization system in a multi-block workspace. Robotica, 28(3):397-403, 2010.

[18] N. B. Priyantha, A. Chakraborty, and H. Balakrishnan. The cricket location-support system. In Proceedings of the 6th annual international conference on Mobile computing and networking, pages 32-43. ACM, 2000.

[19] A. D. Rathnayaka, V. M. Potdar, and S. J. Kuruppu. Evaluation of wireless home automation technologies. In Digital Ecosystems and Technologies Conference (DEST), 2011 Proceedings of the 5th IEEE International Conference on, pages 76-81. IEEE, 2011.

[20] A. Redondi, M. Chirico, L. Borsani, M. Cesana, and M. Tagliasacchi. An integrated system based on wireless sensor networks for patient monitoring, localization and tracking. Ad Hoc Networks, 11(1):39-53, 2013.

[21] A. Redondi, M. Tagliasacchi, M. Cesana, L. Borsani, P. Tarrio, and F. Salice. Laura - localization and ubiquitous monitoring of patients for health care support. In Personal, indoor and mobile radio communications workshops (PIMRC Workshops), 2010 IEEE 21 st International Symposium on, pages 218-222. IEEE, 2010.

[22] G. Retscher. Test and integration of location sensors for a multi-sensor personal navigator. Journal of Navigation, 60(01):107-117, 2007.

[23] H. Saidinejad, J. Radaelli, F. Veronese, and F. Salice. Mixed Technical and Market Evaluation of Home Automation Networks for AAL Solutions, volume Vol. 33: Assistive Technology: From Research to Practice of Assistive Technology Research Series, pages 865 - 870. 2013.

[24] H. Stuckenschmidt, S. Ceri, E. Della Valle, and F. van Harmelen. Towards expressive stream reasoning. In Proceedings of the Dagstuhl Seminar on Semantic Aspects of Sensor Networks, page 241, 2010.

[25] M. A. Sturza. Navigation system integrity monitoring using redundant measurements. Navigation, 35(4):1988-89, 1988.

[26] J. Torres-Solis, T. Falk, and T. Chau. A review of indoor localization technologies: towards navigational assistance for topographical disorientation. Ambient Intelligence, pages 51-84, 2010

[27] H. Wang, S. Sen, A. Elgohary, M. Farid, M. Youssef, and R. R. Choudhury. No need to war-drive: unsupervised indoor localization. In Proceedings of the 10th international conference on Mobile systems, applications, and services, pages 197-210. ACM, 2012.

[28] O. Woodman and R. Harle. Pedestrian localisation for indoor environments. In Proceedings of the 10th international conference on Ubiquitous computing, pages 114-123. ACM, 2008. 